Georgetown University Institutional Repository http://www.library.georgetown.edu/digitalgeorgetown

The author made this article openly available online. Please tell us how this access affects you. Your story matters.

\title{
Visualizing Node Attribute Uncertainty in Graphs
}

N. Cesario, A. Pang, and L. Singh. "Visualizing node attribute uncertainty in graphs." SPIE Conference on Visualization and Data Analysis (VDA). San Francisco, CA: SPIE, 2011.

Collection Permanent Link: http://hdl.handle.net/10822/761517

(C) SPIE Conference on Visualization and Data Analysis

This material is made available online with the permission of the author, and in accordance with publisher policies. No further reproduction or distribution of this copy is permitted by electronic transmission or any other means. 


\title{
Visualizing Node Attribute Uncertainty in Graphs
}

\author{
Nathaniel Cesario ${ }^{1}$, Alex Pang ${ }^{1}$, and Lisa Singh ${ }^{2}$ \\ ${ }^{1}$ Computer Science Department, UC Santa Cruz, Santa Cruz, CA 95064, U.S.A. \\ ${ }^{2}$ Department of Computer Science, Georgetown University, Washington DC 20057, U.S.A.
}

\begin{abstract}
Visualizations can potentially misrepresent information if they ignore or hide the uncertainty that are usually present in the data. While various techniques and tools exist for visualizing uncertainty in scientific visualizations, there are very few tools that primarily focus on visualizing uncertainty in graphs or network data. With the popularity of social networks and other data sets that are best represented by graphs, there is a pressing need for visualization systems to show uncertainty that are present in the data. This paper focuses on visualizing a particular type of uncertainty in graphs - we assume that nodes in a graph can have one or more attributes, and each of these attributes may have an uncertainty associated with it. Unlike previous efforts in visualizing node or edge uncertainty in graphs by changing the appearance of the nodes or edges, e.g. by blurring, the approach in this paper is to use the spatial layout of the graph to represent the uncertainty information. We describe a prototype tool that incorporates several uncertainty-to-spatial-layout mappings and describe a scenario showing how it might be used for a visual analysis task.
\end{abstract}

Keywords: uncertainty visualization, information visualization, graphs, networks, ego networks.

\section{INTRODUCTION}

With the popularity of social networks such as online community networks, publication networks, disease transmission networks, and applications such as bioinformatics, there is a resurgence of interest in graph visualization. Graphs are commonly visualized using a node-link type of display that shows the relationships between nodes and edges. A large part of graph visualization research focuses on dealing with large graphs to reduce clutter and to improving comprehension of the overall structure, for example using a force based layout strategy [1], bundling edges [2], and in combination with distortion mapping [3], etc. (see http://www.cs.umd.edu/hcil/graphvis/ for more examples of recent graph visualization research). Another way that graphs have been visualized is as a matrix with rows and columns representing nodes and matrix entries representing edge values [4]. In these different types of graph visualizations, the focus is on visualizing the relationship of nodes and edges.

The work presented in this paper is different from the core graph visualization research in that our focus is on presenting the uncertainty in the graphs. Including the uncertainty information in graph visualizations is important because what might appear to be strong relationships among a subset of nodes may turn out to be less significant if one factors in node and/or edge attribute uncertainties. For example, the social networking and user review web site provides ratings and comments about local neighborhood establishments such as restaurants, doctors, and so on. The ratings are provided by users who are connected to other users as friends within an online social network context. One can think of the ego network of an establishment as the set of yelp users who have provided a review about it. The establishment node has information about its average rating, and if sufficient ratings are provided, a distribution and a trend chart of ratings as well. Just as one can read the comments about an establishment, one can also read the comments by a particular yelp user. Thus, the user node also has a distribution of ratings he or she has provided. In deciding whether to patronize a particular establishment, one typically uses the average rating as an indicator. What is not available is some measure of uncertainty (or

N.C.: E-mail: ncesario@soe.ucsc.edu

A.P.: E-mail: pang@cse.ucsc.edu

L.S.: E-mail: singh@cs.georgetown.edu 
a measure of trust) about the average rating of the establishment. If each of the user ratings came with an additional attribute field to indicate a trust metric that is factored into the calculation of a weighted average rating for the establishment, the weighted average may significantly differ from the simple average. Such a metric may be based on a number of factors such as usability scores of a particular review, whether the user tends to give high ratings or low ratings, whether the user has enough sample size in the type of establishment being reviewed, and so on. We believe that additional information, such as uncertainty, can lead to better decisions.

There are many types of graphs. One of the most general type is a multi-modal, multi-relational, multifeatured or an $M^{*} 3$ graph [5]. Using the popular internet movie database (http://www.imdb.com/) as an example, multi-modal means there can be more than one type of node, e.g. nodes may represent people or movies; multi-relational means there can be more than one type of edge, e.g. edges may represent different roles that people play in different movies such as a person is an actor in one movie, but is a director or producer in another movie; and multi-featured means there can be more than one attribute for each node or edge, e.g. rating of the movie or the popularity of the actor/actress. Our ultimate goal is to visualize $M^{*} 3$ graphs that contain uncertainty information for each attribute field. The goal of this paper is much more modest as we concentrate on uni-modal and uni-relational but possibly multi-featured graphs where the uncertainty information is available in node attribute fields.

The uncertainty information plays a major role in how we design the graph layouts. This effectively relegates the display of relationships as a secondary criterion, which we address using linked displays [6] and user interaction. Hence, we integrate the visualization methods presented in this paper with the Invenio system [5] to provide user interaction as well as to have access to other functionalities such as data mining. Currently, Invenio can display $M^{*} 3$ graphs without node or edge attribute uncertainties. See Figure 1 for a simple example. Here, a traditional node-link diagram is used and no indications of uncertainty are included. If the data above in fact contained nodes with an attribute called "exists" with an associated uncertainty value, the colored nodes in the picture implies that each node "exists" with total confidence (i.e. zero uncertainty). That is to say, if the nodes in the picture did have this attribute with uncertainty, then the picture is misleading.

In this paper, we investigate different techniques whose primary focus is on revealing the node attribute uncertainty in graphs. We also discuss how these techniques are integrated into Invenio to provide a prototype environment for visualizing such graphs.

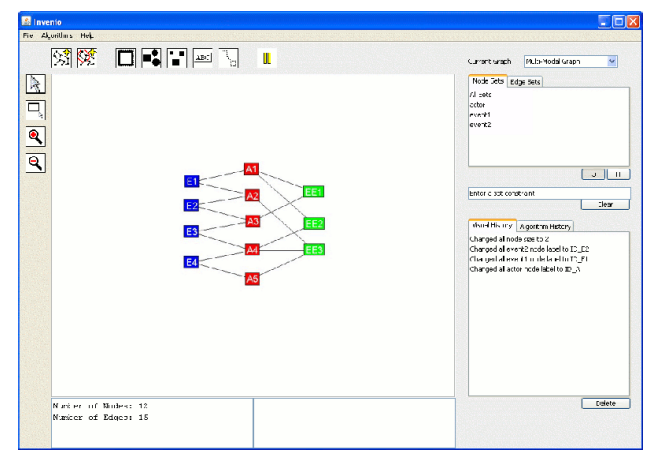

Figure 1. A screenshot of Invenio. Node color represents different types of nodes.

\section{RELATED WORKS}

While there has been little work which focuses primarily on visualizing uncertainty in graphs, there is extensive research in the field for visualizing networks as well as many tools which can be used to visualize a graph or network in a plethora of ways, e.g. force directed layouts, clustering layouts, radial layouts, various tree layouts, etc. An excellent survey of the different graph visualization methods are described in [7] and [8]. In this section, we will briefly discuss some of the research that is related to our research and prototype development. 


\subsection{Lattice Graph Uncertainty}

The work by Collins et al. [9] is one of the few works that addresses uncertainty in graphs. They focus on uncertainties encoded in lattice graphs as exemplified by a case study involving machine translation of a cross language chat application. A sentence is represented as a linear path through a graph structure, where nodes represent words and edges represent the possible connections between words. Multiple linear paths are possible, which are scored according to the likelihood that the right sequence of words is chosen for the translated sentence. Collins et al. mapped this likelihood (or conversely the uncertainty) of a node being the right word to different visual cues such as fuzziness and hue. More importantly, they also redundantly encoded uncertainty to the vertical position of the node (see Figure 2). It is this latter positional mapping of uncertainty that we wish to explore further in this paper. Specifically, we explore alternative graph visualization methods where the uncertainty information is the primary driver for determining the spatial layout of the graph.

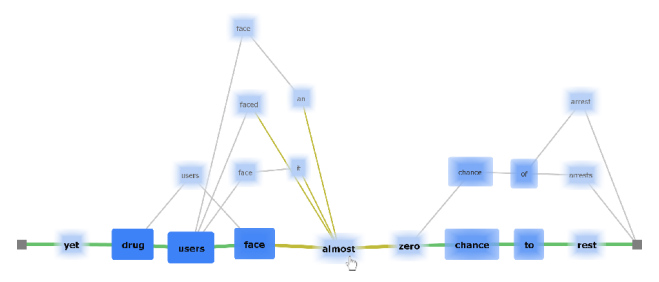

Figure 2. Node fuzziness used to display uncertainty [9]. Vertical positions of nodes also represent uncertainty.

\subsection{Visone}

Visone, created by Brandes and Wagner [10], is a tool designed specifically to explore and analyze social networks. It can analyze attributes attached to nodes and edges that may represent probability. While visone provides many useful graph representations for viewing node and edge attributes, it is not possible, to our knowledge, to view multiple representations of a particular graph with linked coordinated views.

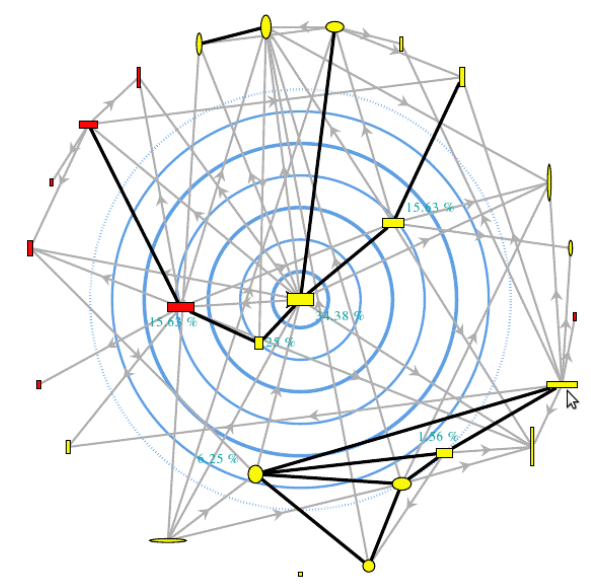

Figure 3. A radial visualization using Visone showing the betweenness metric of organizations involved in drug policy making. Edges can be "confirmed" or "unconfirmed" as denoted by their color while different node attributes are mapped to the shape, color, width, and height of nodes.

\subsection{Jigsaw}

On the other hand, there are some very nice information visualization systems that are geared towards visual analytics by providing users the ability to interact, perform drill downs, and obtained linked coordinate views, etc. Jigsaw is one such system. It is a tool for investigative analysts to explore textual data [11]. Multiple 
displays are also used to give the user multiple views of the data which include highlighted textual views, list views, and traditional node-link diagrams.

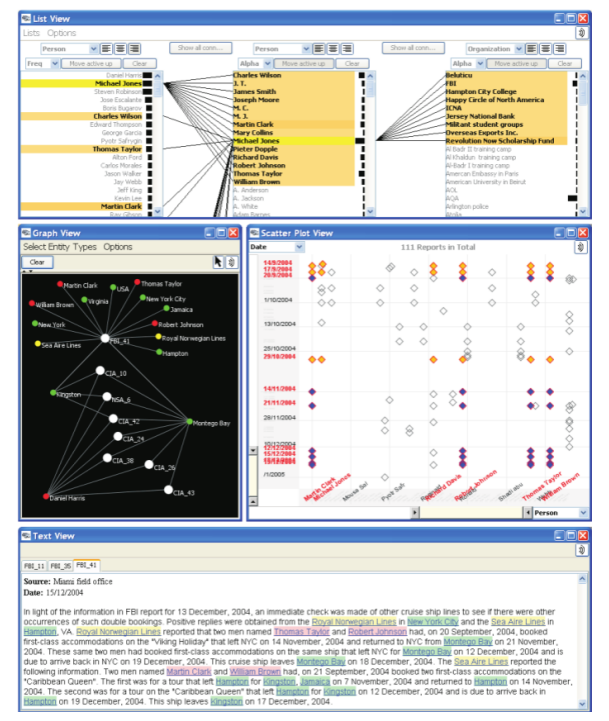

Figure 4. An example of some of the visual techniques used in Jigsaw for visualizing textual data for investigative analysts.

In Figure 4, an analyst is investigating a "suspicious" person and can use a list view (top) to sort elements and view associations between people and networks, a node-link diagram (middle-left) to view people associated with reports, a scatter plot view (middle-right) to show when reports were filed and by whom, and a textual view (bottom) highlighting important terms in a report. While Jigsaw is intended mainly for textual data, this example shows how it can be used to display different attributes in network data.

\subsection{Invenio}

Singh et al. [5] developed a tool called Invenio for exploring $M^{*} 3$ networks (see Figure 1). It combines a wide variety of visualization from Prefuse [12] and data mining algorithms from Jung [13] to allow users to explore $M^{*} 3$ networks. Because of these capabilities and our longer term goal of visualizing uncertainty in $M^{*} 3$ graphs, we have integrated the methods described in this paper into Invenio.

\section{IMPLEMENTATION}

Since we are integrating our work into Invenio and since Invenio uses Prefuse, it is natural that we write our tool using Prefuse (release 2007.10.21). Prefuse [12] is a toolkit written in Java by Jeffrey Heer at Stanford University. It uses the Java2D API for rendering.

Since Prefuse is designed to display graph data, much of the functionality we needed for displaying our data is already implemented. Prefuse also uses a table data structure to store nodes and edges. This is ideal for working with $M^{*} 3$ networks as it allows us to attach an arbitrary number of attributes to nodes and edges, including an uncertainty field for each attribute. Since the elements in the table data structure are references to Java objects, the attributes for nodes and edges can be arbitrarily complex or simple.

We are primarily using screenspace and manipulating the position, shape, color, opacity, etc. of nodes and edges. Prefuse abstracts these features in such a way that allows us to more or less concentrate entirely on the visual features we are interested in. 


\section{THE PROTOTYPE}

Our prototype tool consists of interaction and graph layout components combined to facilitate the experimentation of new ideas for visualizing uncertainty in graphs. As described earlier, the layouts used in the visualizations are primarily designed to highlight the uncertainty information in the data. The interaction components allow the users to examine the data in more detail via linked coordinated views.

\subsection{Spatial Layouts Based on Uncertainty}

We will first introduce each visual layout used in our prototype giving a brief explanation and example of each.

\subsubsection{Bullseye}

The concept of the bullseye is as follows: given $n$ graphs (or ego networks), split the bullseye into $n$ "graph sectors". Furthermore, if nodes in the graphs have $s$ different attributes, we will split each "graph sector" into $s$ "sub-sectors." At the moment, we have only concentrated on instances where $n=2$ and $s \leq 5$. Since a bullseye is a polar mapping, we can theoretically place an infinite number of graphs on the bullseye with each graph having an infinite number of attributes (where infinite is taken to be "practically large"). However, this layout will get cluttered rather quickly as we increase the number of graphs or attributes since the graph sectors and sub-sectors will become very small. Still, having a very large number of graphs and/or attributes can still be useful especially when coupled with brushing or filtering techniques.

Once we find out which sector and sub-sector a node belongs to, we then determine its position within the sector. Using polar coordinates, where $\theta$ and $r$ are the angular and radial components respectively, we will map $\theta$ to a particular attribute's value, and $r$ to the confidence (or 1 - uncertainty) of that attribute's value where $\theta$ is linearly interpolated across the sub-sector: $\theta \in$ (start of sub-sector, end of sub-sector).

The radial component, $r$, will not necessarily be a value from 0 to $R$ where $R$ is the outer radius of the bullseye. Instead, the bullseye can have "rings." Rings can be used for different levels of confidence. They can also be used to show which nodes match or don't match between graphs or ego networks. Each ring is demarcated by an inner and an outer radius: $r_{a_{i}}$ and $r_{a_{o}}$ respectively. Note that these two radii are not required to have similar values. For example, $r_{a_{i}}$ can be set to 0 if one is not interested in doing comparisons. Given a node with attribute uncertainty value of $u_{n}$, then the radial component of this node's position is obtained via linear interpolation: $r_{n}=u_{n}\left(r_{a_{o}}-r_{a_{i}}\right)+r_{a_{i}}$.

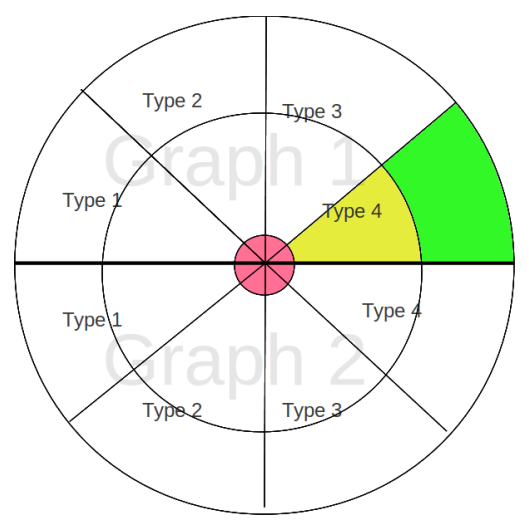

Figure 5. Descriptive diagram of the bullseye layout.

Figure 5 displays a bullseye split into an upper and a lower half to simultaneously show 2 graphs. Each graph has 4 node attribute types, e.g. actors, actresses, directors, producers. This particular bullseye has two rings. The green and yellow regions denote portions of the outer and inner rings respectively. The optional shaded disc in the center allows a minimum threshold to be set for probability values.

Such a layout can be used to compare two ego networks (which can be sub-graphs of the same graph) as follows: nodes that are common neighbors of both ego networks are placed in the inner ring, while nodes that 
are not shared by both ego networks (including the 2 nodes whose ego networks are being compared) are placed in the outer ring. The utility of such a display is less on seeing the relationships among nodes, but rather to see if there is some pattern in the distribution of attribute values and attribute uncertainty values. More formally, in set notation, a graph is defined as $G=(V, E)$ containing a set of nodes, or vertices, $V$ and connected by a set of edges $E$. Suppose $v_{a}$ and $v_{b}$ are two egos in $G$, then their respective ego networks form sub-graphs $G_{a}$ and $G_{b}$. The nodes in each of these sub-graphs, $V_{a}$ and $V_{b}$, are nodes in $G$ with direct connections to $v_{a}$ or $v_{b}$ respectively. If $M$ represents the set of nodes in the inner ring and $m$ represents those in the outer ring, then $M=\left\{V_{a} \cap V_{b}\right\}$, and $m=\left\{\left(V_{a}-M\right) \cup\left(V_{b}-M\right)\right\}$. If no ego networks have been selected for comparison, as in the initial state of our prototype, then all nodes appear in the outer ring.

\subsubsection{Comparative Column}

The comparative column (CC) display is designed for comparing two ego networks or graphs with node attribute uncertainty. A simple 2D rectangular space is used to layout node attribute uncertainties. An important aspect of the layout is the ability to distinguish the relative uncertainty values of corresponding nodes between the two graphs. Figure 6 illustrates the general concept. This layout can be used to display one attribute uncertainty field at a time. For instance, it can be used to compare a particular node attribute uncertainty between the two graphs with uncertainty. If the node attribute from the two graphs have different uncertainty values, then that node will be positioned horizontally closer to either the left or the right edge of the rectangular space accordingly. If the node attributes are quite similar, say both have uncertainty values of 0.3 , then the node is placed at the center along the horizontal axis. The vertical axis is used to display the combined uncertainty values. Thus, the nodes towards the top of the rectangle have a lower combined uncertainty, and nodes towards the bottom have a higher combined uncertainty. The node attribute uncertainties can be combined by simply taking their average uncertainty, or some other measurement of overall confidence can be used.

Unlike the bullseye layout, a common node is displayed only once in the CC layout. Nodes that are in one graph but not the other are not displayed in a CC layout. Therefore, one can use the CC layout as a means to extract further detail about nodes in an inner ring of a bullseye layout.

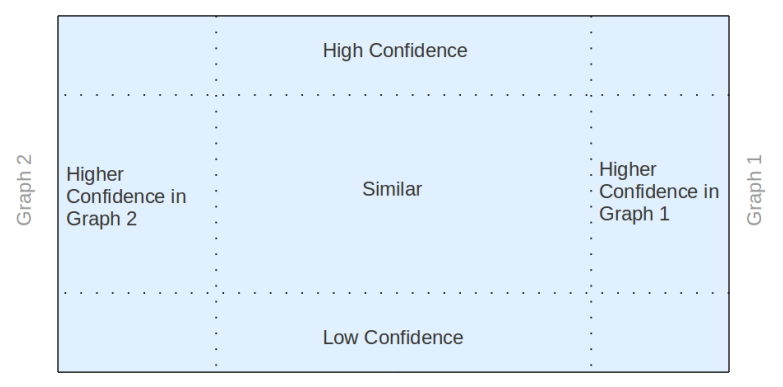

Figure 6. Diagram showing the meaning of each area in the comparative column view.

\subsubsection{Scatter Plot}

This is a traditional scatter plot between an attribute value and the attribute uncertainty value. Because nodes may have multiple attributes, one can view each attribute as being a separate scatter plot stacked on top of each other as illustrated in Figure 7. This aspect is similar to the sub-sectors in a bullseye layout.

\subsubsection{Fisheye Filter}

With any of the aforementioned techniques, the display can become cluttered when there are many nodes to visualize. One way to combat this is to use something similar to a fisheye lens described in the review by Leung and Aerley [3]. We slightly modified the fisheye algorithm provided by Prefuse in order to create a "targeted fisheye" which only sees nodes of a particular attribute type at the focal point while nodes of the non-target attribute type disappear at the focal point, but are still visible in the outer edges of the fisheye. We accomplish this by making nodes of the non-target attribute type not visible if they are within a certain distance threshold from the focal point of the fisheye. More precisely, if $f=(x, y)$ is the focal point, $d$ is the distance threshold, 

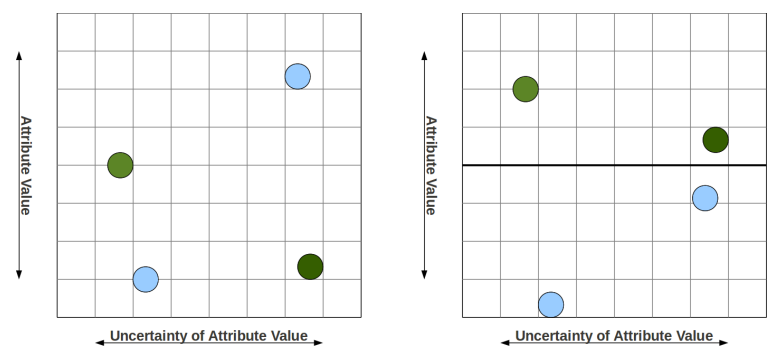

Figure 7. Example of the scatter plot view with (a) different node attributes intermixed (different types are colored differently), and (b) node attributes separated into vertical "bins" of different types.

and $\alpha$ is the target value for node attribute $t$, then

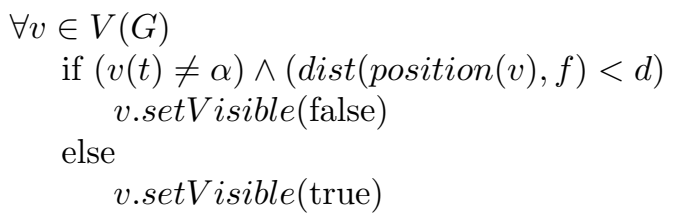

where $v(x)$ is the value of attribute $x$ for vertex $v$. Note that rather than using a strict cut-off for visibility determination, an alternative is to use alpha blending.

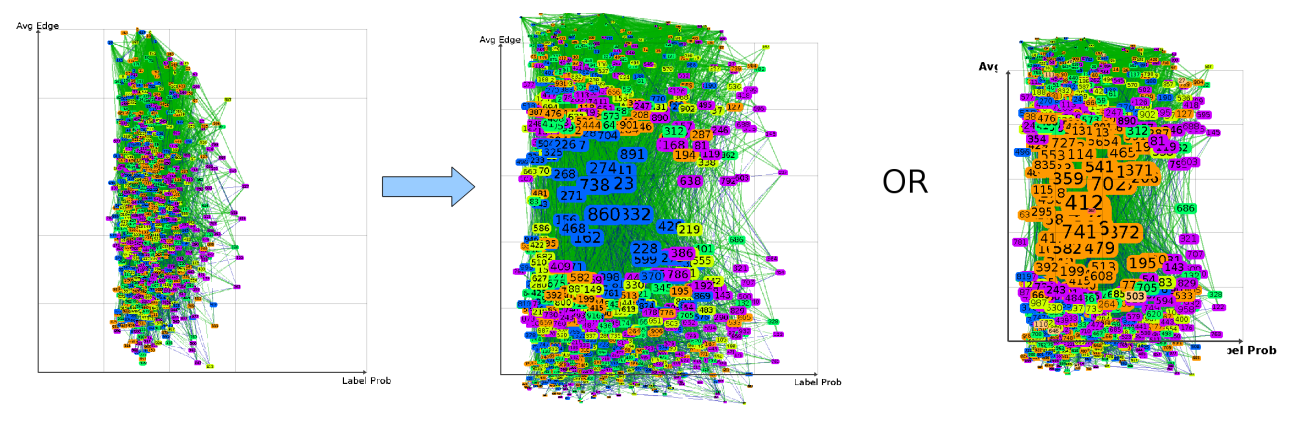

Figure 8. An example of the targeted fisheye. Only blue nodes appear near the center of the fisheye's focal point. x-y positioning of nodes is only preserved at the focal point of the fisheye. Node color is mapped to the label attribute, so in this case we are targeting nodes such that $v($ label $)=$ blue (middle) and $v($ label $)=$ orange (right).

\subsubsection{Parallel Coordinates}

Parallel coordinates [14] are very useful when it comes to visualizing multidimensional data. In the case of graphs with node attribute uncertainty, we can treat the uncertainty of each attribute as a dimension in a multidimensional space. Figure 9 shows an example with three person nodes (Bob, Jane, and John), each of which has three attributes: age, gender, and profession along with their associated uncertainty values. The attribute uncertainties are the variables of interest and are displayed as separate axes. The uncertainty values indicates the confidence level of the attribute value. This view tells us we are very sure of Bob's age, the gender of all three people, and John and Jane's profession while we are not so sure about John's age and Bob's profession. Note that earlier work by Haroz et al. [15] also use a similar mapping for visualizing uncertainty in scientific data sets.

\subsection{Linked Views and User Interactions}

Each of the visual techniques mentioned in this section gives a drastically different view from one another and, with the exception of parallel coordinates and the bullseye layouts, are designed to only display and compare one 


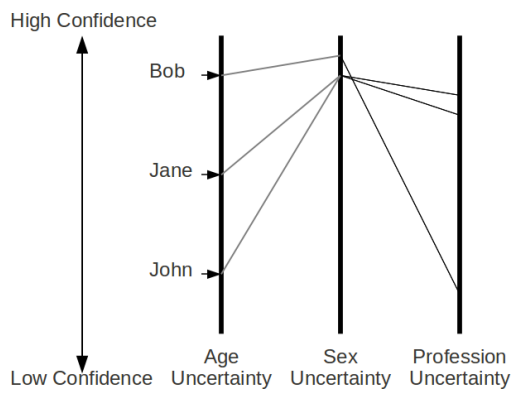

Figure 9. Parallel coordinates used to visualize node attribute uncertainty.

or two attributes. Since nodes may often contain tens of (if not more) attributes, our tool needs the ability to have many displays with the option to link them together in order to make these many different "vantage points" of the data available to the user. Figures 10 to 14 illustrate how this might be accomplished with multiple views.

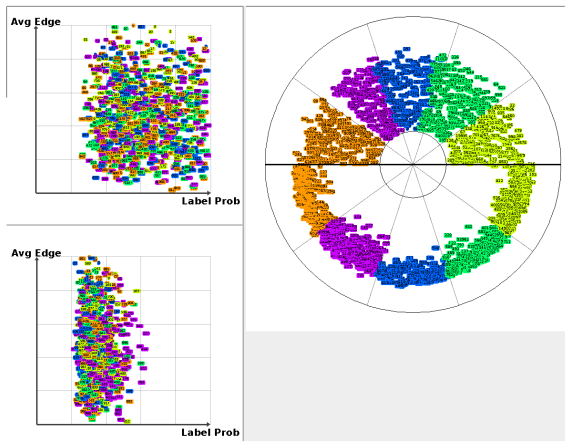

Figure 10. An example of linked displays in our prototype. Here we want to compare 2 graphs, each with 5 node attributes and their uncertainty. The scatter plot on the top left shows that this graph's distribution of uncertainty values are skewed to the right, but the attribute values themselves are uniformly distributed. The scatter plot on the lower left shows that the second graph's uncertainty values are skewed to the left and has a smaller spread. The bullseye on the right shows an alternative way to view the distribution of uncertainty values. Note that the inner ring is not used in this example. Nodes in both the bullseye and scatter plots are colored by attribute type, and edges are hidden to help reduce clutter.

\section{SAMPLE SCENARIO}

For a sample scenario, we consider a case where a breeder is doing some exploratory analysis on the inbreeding within Otterhounds and hip dysplasia, which is associated with abnormal joint structure and a laxity of the muscles, connective tissue, and ligaments that would normally support the joint [16]. To do this, we calculate the coefficient of inbreeding (COI) [17]. The coefficient of inbreeding can be thought of as a measure of how closely related the parents of a particular dog are. There are many algorithms for calculating the COI [18] which will not be covered here. For our purpose, we use the simplest method, or paths method. In Figure 15, we examine our test data using parallel coordinates to view the gender, hip scores, color, and COI, and we complement this view with Prefuse's force-directed node-link layout. The hip score is a measure of how bad hip dysplasia is for a particular dog, from a severe case of dysplasia to excellent hips (no dysplasia). The visible edges in the node-link layout are directed such that the child node points to the parent node. In Figure 15 we select only hips that would be classified as "poor" to "severe" and notice that gender, color, and inbreeding appear to have no bearing on low hip scores since the subset of dogs we have selected have a broad range in gender and color and a $C O I$ of 0 . However, the further we can trace back a dog's lineage, the more likely it is that some inbreeding occurred (operating on the notion that if we go back far enough in time there was a 


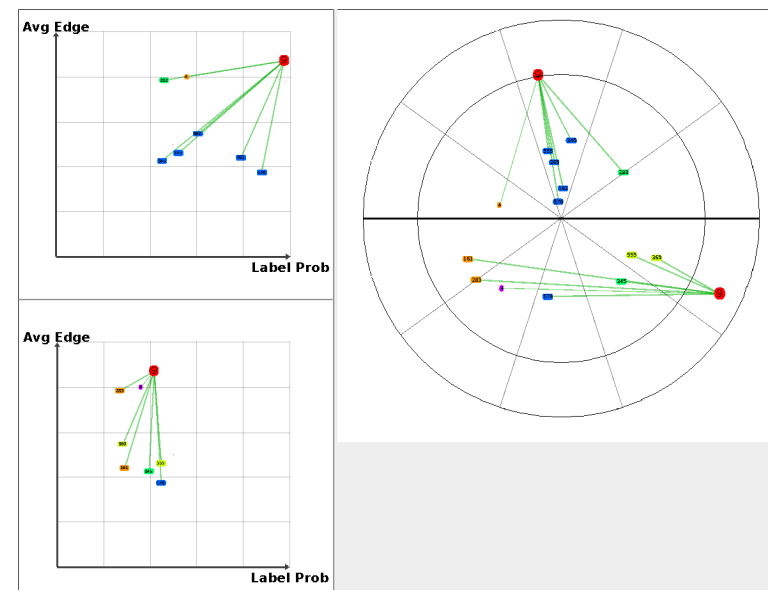

Figure 11. Comparing ego networks from the same pair of graphs from Figure 10. By clicking on a node (in this case node "9"), we compare its ego network in the top left graph (graph 1) with the corresponding ego network of node "9" in the bottom left graph (graph 2). Since the ego networks of node "9" in both graphs are identical, all nodes appear in the inner ring. The two nodes appearing in the outer ring (one on the top half, one on the bottom half) represent node "9."

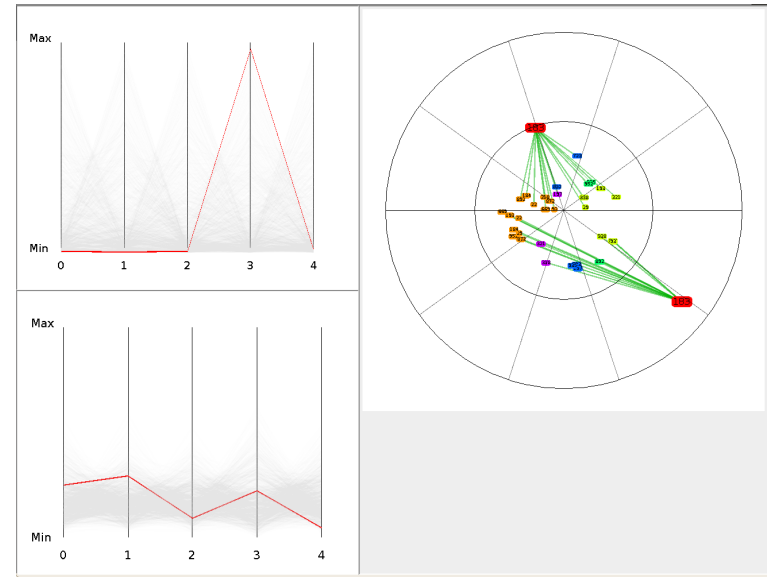

Figure 12. Using the same graphs as in Figure 10, the scatter plot is replaced with the parallel coordinate view where the axes represent different node attribute uncertainty. In this particular instance, we have selected a node from the top graph by selecting this node's corresponding poly-line in the parallel coordinates view. The ego network of the selected node is also highlighted in the bullseye layout.

smaller population of dogs). We handle incomplete or missing information in the following manner: for a given dog, we generate probabilities based on how much knowledge we have about his or her lineage. We look at up to 4 generations of a dog's lineage, and assign $25 \%$ probability to each generation. So each parent gets $12.5 \%$ (times 2) probability, each grandparent gets $6.25 \%$ (times 4), each great grandparent gets $3.125 \%$ (times 8), and each great, great grandparent gets $1.5625 \%$ (times 16). For example, if we know one parent, two grandparents, and three great grandparents in a particular dog's lineage, we say that the probability the COI for that dog is correct is $12.5 \%+2(6.25 \%)+3(3.125 \%)=34.375 \%$.

This leads us to Figure 16, where we highlight the same set of nodes, and replace the force-directed view with a scatter plot and bullseye views to provide additional information about uncertainty. In the scatter plot view, we are comparing hip scores to the probability our COI calculation is correct. While in the bullseye view, we are just looking at the probability the $C O I$ is correct (nodes closer to the center have a higher chance of being correct). The selected nodes, highlighted in the selection box in the parallel coordinates view, are shown as larger nodes in the two other views. These linked views suggest that, while all of the selected dogs with very 


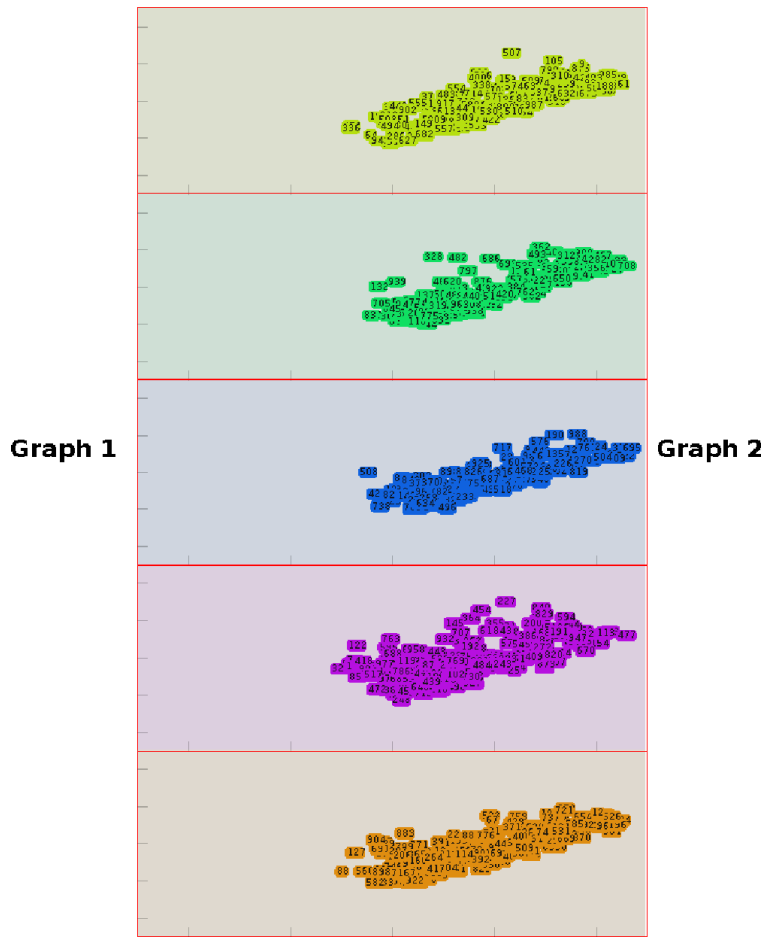

Figure 13. The same data set as in the previous figures are shown using the $\mathrm{CC}$ view. The fact that the majority of nodes are shifted to the right means that most of the nodes have less uncertainty in graph 2 . In this particular view, we are actually using $5 \mathrm{CCs}$, one for each node attribute.

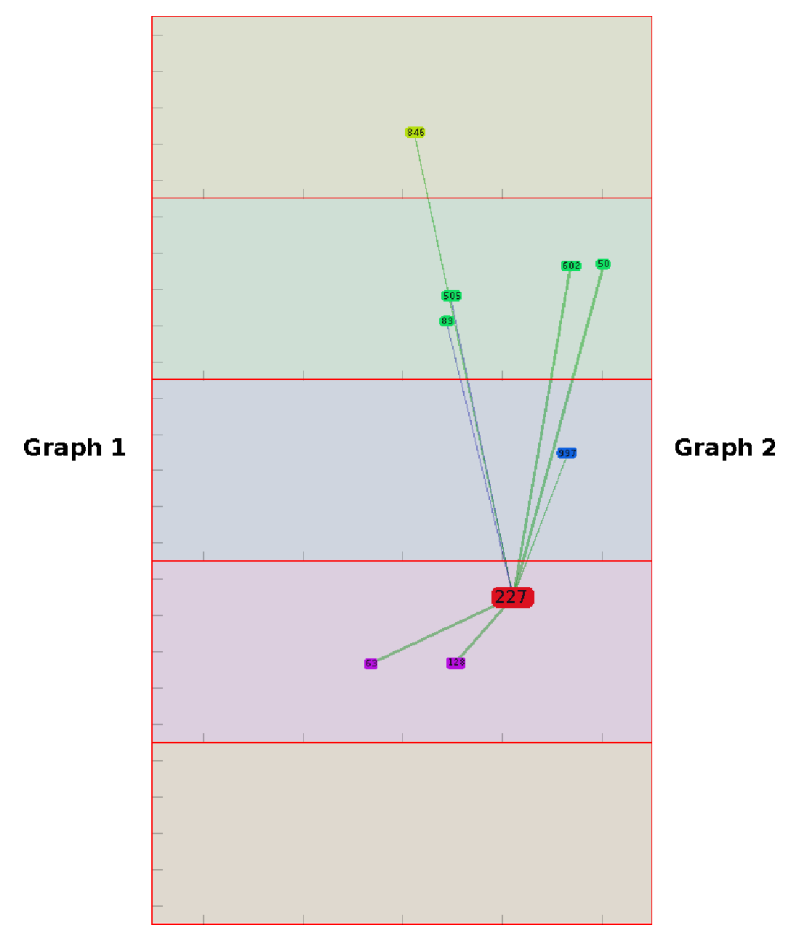

Figure 14. Using the CC view to look at the ego network of a node. We can see that nodes within this ego network are fairly split having either a higher confidence in Graph 2 (nodes towards the right of the column), nodes that have about the same confidence in both graphs (nodes in the center), and one node that has slightly higher confidence in Graph 1.

low hip scores also have a low $C O I$ value, the probability of the $C O I$ value being correct is actually quite low (less than 20\%).

Having the scatter plot and parallel coordinates views linked allows us to explore what impact a particular attribute (e.g. color) has on two variables (e.g. COI probability and hip score) by selecting them in the parallel coordinates view and viewing the results in the scatter plot view. For example, colors that are mostly black have a lower index value than those that are mostly white. If we select the group of $\operatorname{dogs}$ with the highest $C O I$ from the parallel coordinates view, we can see that no dogs that are mostly white are selected (figure omitted due to page limit). This could lead a researcher to perhaps compare color to other attributes he or she would not have thought of before. Because this is a dataset with a small number of attributes, we found the parallel coordinates view to be mostly sufficient by itself. The bullseye view was not particularly helpful here because it is used to compare three or more graphs or compare ego networks within a graph that has 3 or more node types. In this dataset there were only 2 types of nodes: male and female.

This example is used only as a simple illustration. We hope to publish future results using the comparison features of our visualizations looking at datasets with many more attributes.

\section{DISCUSSION}

During the course of this research, there are a number of issues that are worth mentioning. 

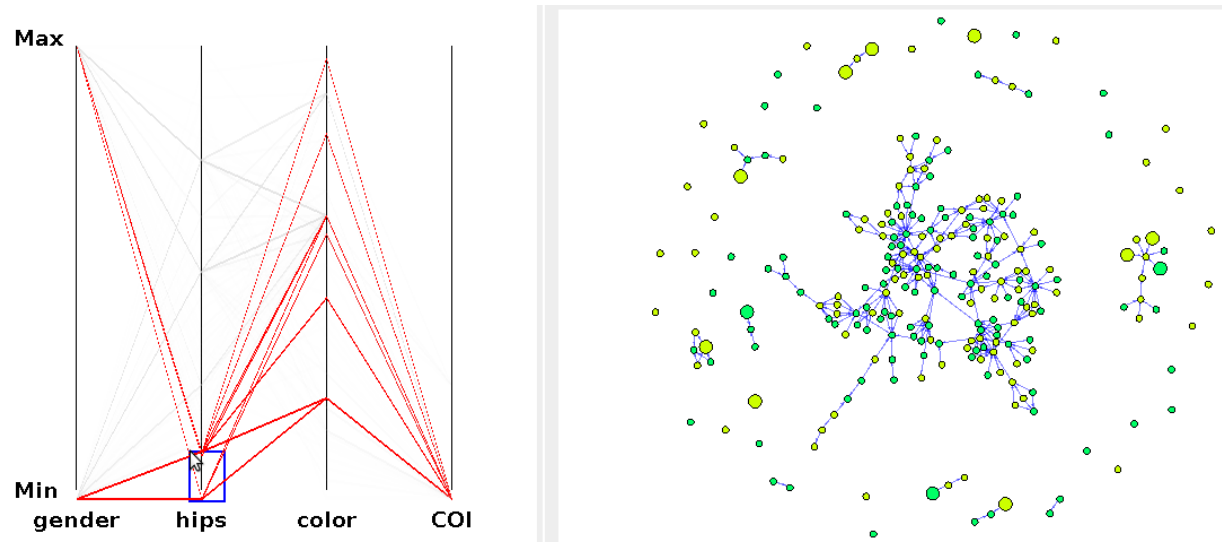

Figure 15. A look at a subset of the Otterhound database using parallel coordinates (left) and a force-directed layout (right). Each node shown in the force-directed view is also represented as a polyline on the left. The edges in the force-directed view are directed such that a child points to its parents.

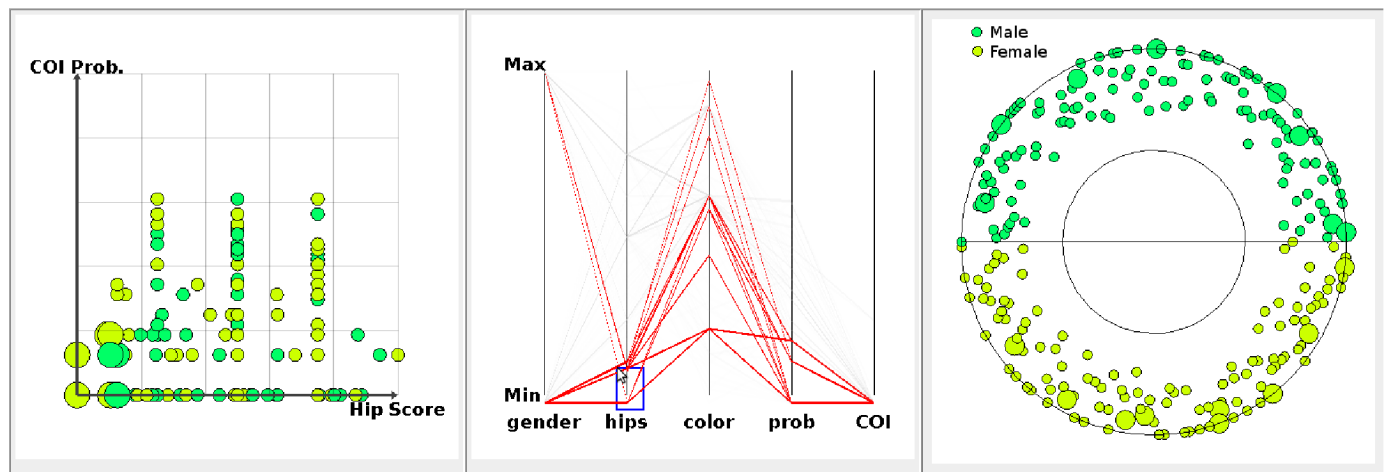

Figure 16. The same Otterhound data as in Figure 15. Dogs that are selected by the blue box in the parallel coordinates view are shown as larger nodes in the scatter plot and bullseye views.

\subsection{Visualizing Edge Types and Attributes}

Most of the work to date have focused on node attribute uncertainty. For edge attribute uncertainty, we have mainly used line thickness, color, and opacity of edges to represent uncertainty. However, as is apparent in Figure 8 , these mappings are not useful when there are many edges. We are exploring the use of the background space where the graph is layed out to provide additional information about the node and edge attribute uncertainties.

\subsection{More Interactivity}

Currently, the number of linked displays are set to three views. This is mainly based on our observation that having more than three simultaneous views made it difficult to track changes from one view to another. However, we have not investigated what the ideal number of linked displays should be. Lacking that, the user should be able to add as many displays as he or she wants, or as many as is suitable for his or her screenspace. The user should be able to experiment with all techniques, simultaneously if desired, and remove displays which do not appear to be beneficial.

While linked displays can be very useful, there may be cases where the user wishes to explore different parts of a dataset in conjunction with the multiple displays of some other parts of the same dataset. This is currently not possible as all displays are linked by default. Therefore, whatever data is being examined in one display is also the focus of all the other displays. There may be instances where having the option to unlink displays is beneficial. 


\subsection{Details-on-Demand}

As can be seen in many of the example figures, the displays can become cluttered quite easily and it is often desirable to display a single node as a simple shape with no information, except for color, perhaps. In the example images displayed here, notice that there is only a number (the node's ID) displayed on each node, there are actually hundreds of attributes associated with each node. For this reason, the user should be able to immediately see all or a large portion of this data upon selecting a node.

\subsection{Animation}

While the prototype is interactive, nothing is animated. It would be helpful to give the user contextual information when he or she changes the display. For example, if the user changes the layout used in a display, if nodes "migrate" to their new positions rather than just appear there, it would give the user an idea of how one layout relates to another and possibly help them to understand the data better.

\subsection{Need for User Evaluation}

As the integration with Invenio is stabilized, we will have a platform for exploring new visual layouts. There is a need for user evaluations of which ones work best for different situations, as well as a need for user evaluations of the interaction environment. In addition, it would be helpful to look at data which contains uncertainty and then view it using traditional tools which do not take uncertainty into account and contrast those results with results from using our tool.

\section{CONCLUSION}

This paper reports on our initial attempts at visualizing graphs with node attribute uncertainty using a combination of linked views, user interactions, and 5 different layouts, namely: bullseye (4.1.1), comparative column (4.1.2), scatter plot (4.1.3), fisheye (4.1.4), and parallel coordinates (4.1.5). The major design variable for the different layouts was to show the uncertainty, while showing the relationships amongst the nodes played a secondary role. We believe that integration of the tools into Invenio will facilitate exploration of other alternative visualization methods for visualizing uncertainty in graphs. Finally, while the current prototype can be used to view multi-featured graphs with uncertainty, more research needs to be done to simultaneously show more information, i.e. node and edge attribute uncertainties together, graph relationships, and full $M^{*} 3$ graphs with uncertainty.

\section{ACKNOWLEDGMENTS}

This work is supported by the National Science Foundation under Grant No. 0937073. We thank the anonymous reviewers for their constructive comments and suggestions.

\section{REFERENCES}

[1] Fruchterman, T. M. J. and Reingold, E. M., "Graph drawing by force-directed placement," Software: Practice and Experience 21(11), 1129-1164 (1991).

[2] Holten, D., "Hierarchical edge bundles: Visualization of adjacency relations in hierarchical data," IEEE Transactions on Visualization and Computer Graphics 12, 741-748 (September 2006).

[3] Leung, Y. K. and Aerley, M. D., "A review and taxonomy of distortion-oriented presentation techniques," ACM Transactions on Computer-Human Interaction 1(2), 126-160 (1994).

[4] Ghoniem, M., Fekete, J.-D., and Castagliola, P., "A comparison of the readability of graphs using node-link and matrix-based representations," in [INFOVIS '04: Proceedings of the IEEE Symposium on Information Visualization], 17-24, IEEE Computer Society, Washington, DC, USA (2004).

[5] Singh, L., Beard, M., Getoor, L., and Blake, M. B., "Visual mining of multi-modal social networks at different abstraction levels," in [IV '0\%: Proceedings of the 11th International Conference Information Visualization], 672-679, IEEE Computer Society, Washington, DC, USA (2007). 
[6] Stolte, C. and Hanrahan, P., "Polaris: A system for query, analysis and visualization of multi-dimensional relational databases," in [INFOVIS '00: Proceedings of the IEEE Symposium on Information Vizualization 2000], 5, IEEE Computer Society, Washington, DC, USA (2000).

[7] Herman, I., Melancon, G., and Marshall, M. S., "Graph visualization and navigation in information visualization: A survey," IEEE TRANSACTIONS ON VISUALIZATION AND COMPUTER GRAPHICS 6(1), $24-43(2000)$.

[8] Cui, W., "A survey on graph visualization," (2007). http://www.cse.ust.hk/ weiwei/PQE/WeiweiPQE.pdf.

[9] Collins, C., Sheelagh, and Penn, G., "Visualization of uncertainty in lattices to support decision-making," in [EuroVis], Museth, K., Möller, T., Ynnerman, A., Museth, K., Möller, T., and Ynnerman, A., eds., 51-58, Eurographics Association (2007).

[10] Brandes, U. and Wagner, D., "visone - analysis and visualization of social networks," in [Graph Drawing Software], 321-340, Springer-Verlag (2003).

[11] Gorg, C., Liu, Z., Parekh, N., Singhal, K., and Stasko, J., "Visual analytics with Jigsaw," in [VAST '07: Proceedings of the 2007 IEEE Symposium on Visual Analytics Science and Technology], 201-202, IEEE Computer Society, Washington, DC, USA (2007).

[12] Heer, J., "Prefuse: information visualization toolkit." http://prefuse.org.

[13] JUNG framework development team, "JUNG: Java Universal Network/Graph framework." http://jung.sourceforge.net.

[14] Inselberg, A. and Dimsdale, B., "Parallel coordinates: a tool for visualizing multi-dimensional geometry," in [VIS '90: Proceedings of the 1st conference on Visualization '90], 361-378, IEEE Computer Society Press, Los Alamitos, CA, USA (1990).

[15] Haroz, S., Ma, K.-L., and Heitmann, K., "Multiple uncertainties in time-variant cosmological particle data," in [IEEE Pacific Visualization Symposium], 207-214 (2008).

[16] Foster, D. and Staff, E., "Hip dysplasia in dogs: Diagnosis, treatment, and prevention." http://www.peteducation.com/article.cfm?c=2+2084\&aid=444.

[17] Wright, S., "Coefficients of inbreeding and relationship," American Naturalist 56, 330-338 (1922).

[18] Sargolzaei, M. and Iwaisaki, H., "Comparison of four direct algorithms for computing inbreeding coefficients," Animal Science Journal 76(5), 401-406. 